\title{
A Tilting Air-Lubricated Piston Gage for Pressures Below One-Half Inch of Mercury ${ }^{1}$
}

\author{
U. O. Hutton
}

(May 8, 1959)

\begin{abstract}
A description is given of a tilting dead-weight piston gage constructed at the National Bureau of Standards for ranges of differential pressure up to about 0.5 inch of mercury. A resolution of better than 1 part in a hundred thousand of full scale has been obtained by use of the toolmaker's sine bar method of angle measurement. The scale is a linear function of the sine. The instrument can be calibrated from basic measurements of length and weight, is rugged, and may be constructed in almost any laboratory mechanical shop. Sources of possible errors in reading are discussed in detail. Comparative tests with certain other gages or manometers are cited wherein linearity was found to be within 1 part in 10,000 and agreement within 2.5 parts in 10,000 . The uses of the gage are briefly discussed.
\end{abstract}

\section{Introduction}

A need exists for pressure-measuring or controlling devices for ranges of low pressure, particularly the pressure range corresponding to atmospheric pressure at high altitudes. The need is more acute where measurement and control are both required. For the study of the pressure-deflection characteristics of diaphragms of high sensitivity, no device discussed in available literature [1] seemed conveniently applicable to the problem where constancy of pressure difference from a datum was desired for various lengths of time up to $24 \mathrm{hr}$. Conventional airlubricated piston gages are nicely applicable to this problem approaching within about 0.4 in. of mercury of the datum pressure with a resolution of at least 1 part in 10,000 . This report describes a piston gage to cover from about $0.5 \mathrm{in}$. of mercury to the datum pressure with a resolution of 1 part in 100,000 and means of pressure adjustment without resort to weight changing.

In exploring the characteristics of gages suitable for the contemplated tests, several air-lubricated piston gages were made from medical hypodermic syringes of 25 - and $100-\mathrm{cm}^{3}$ capacity. These syringes had hollow pistons and could be employed to as low a pressure as $0.4 \mathrm{in}$. of mercury. Schemes for employing such gages with opposed pistons were abandoned because of the loss of resolution as the pressure difference set up by the pistons becomes small. In handling a well-lapped syringe it was observed that air lubrication for a low-density piston was surprisingly adequate even if the piston was not vertical. Brubach's experiments [2] encouraged an attempt to devise a piston gage, the incremental loading of which would be adjusted by changing the angle of tilt instead of the weights as on the conventional dead-weight testers. Distinct advantages of this design are that it reaches high resolution without

1 The major part of this work was sponsored by the Flight Control Laboratory, Wright Air Development Center, Wright-Patterson Air Force Base, Ohio. resort to delicate structures and requires neither optical nor electrical amplification. The extended scale available is gained through the use of a micrometer and end gage block.

\section{Description of Piston Gages}

In development, two models were constructed that will be referred to as Models I and II. Model II incorporates all the features of Model I so that a discussion of Model I is possibly not of great value; however, its simplicity of construction and the high sensitivity and resolution attained may make it applicable in some cases where the additional complexity of Model II is not warranted. The first model is of such simplicity that it may be constructed and a basic calibration made in a short time in almost any laboratory. The principles of the gage are so elementary that adherence to the details being described is not suggested as being necessary. Dimensions and other specific details as used are included for guidance in understanding the structures on which performance data is presented.

\subsection{Description of Gage, Model I}

For the piston-cylinder combination, four $100-\mathrm{cm}^{3}$ syringes were obtained. Two of these were found to be sufficiently tight for adaptation. They were put through a hand-lapping operation where lapping powder, Linde $\mathrm{A}(1 / 2 \mu)$, was used in a creamy water suspension. From $1 / 2$ to $2 \mathrm{hr}$ lapping was employed using a combination of rotary and longitudinal motion with the addition of water or lapping compound as required. The lapping operation was considered complete when a piston, washed and dried, would just fall of its own weight in its cylinder oriented in a vertical position with the piston at any radial angle. It does not seem possible to overlap such a combination with this size of lapping particle using water as a lubricant because of the hydrodynamic lubrication furnished by the lapping motion; however, it was always possible to remove the high areas on the 
piston and cylinder so that the freedom-of-fall condition described was obtained. The tighter areas may be recognized while lapping by the degree of dispersion of the lapping compound and after drying by the higher grade finish on the closer surfaces. Extra attention may be given the high areas by localized finger pressure on the cylinder and by choice of the piston orbit to bring them into frequent play. In some of the better lapped areas, white light interference fringes have been visible but this is not necessary for proper performance.

After lapping, the cylinder and piston were cut with a diamond saw as shown in figure 1 , then mounted on a sine bar, shown in figure 2 to form a piston gage. The piston is weighted as desired and floats without rotation in the cylinder on an air film as a bob. The cylinder is supported on another portion of the original hypodermic piston employed as an air-lubricated pinion bearing. The micrometer anvil and sine pivot are at such a relative height that the piston is horizontal when the micrometer is fully retracted.

The cylinder must be rotated to keep the piston free. It was originally rotated by an air jet directed against the teeth of a 4 -in. diam spur gear concentrically mounted on the cylinder, but later by a $25-\mathrm{w}$, 2,700-rpm shaded-pole induction motor operated at $1 / 3$ voltage with a rubber band $\left(\mathrm{No}_{0} 32\right)$ as a belt over the motor shaft and around the cylinder. A slight finger spin is sometimes required for starting, and under the light load conditions prevailing, the rubber band, as a belt, lasts several weeks and vibration or temperature transmissions from motor to cylinder are negligible.

The piston is eccentrically loaded to prevent its rotation as shown in figure 2 . One inch of vertical travel of the micrometer corresponds to 40 revolutions of the micrometer screw. The length of the scale on the barrel of the micrometer is $1.5 \mathrm{in}$., which gives a total scale length for 40 revolutions of 60 in., with a readability of 0.005 -in., corresponding to 1 in 10,000 .
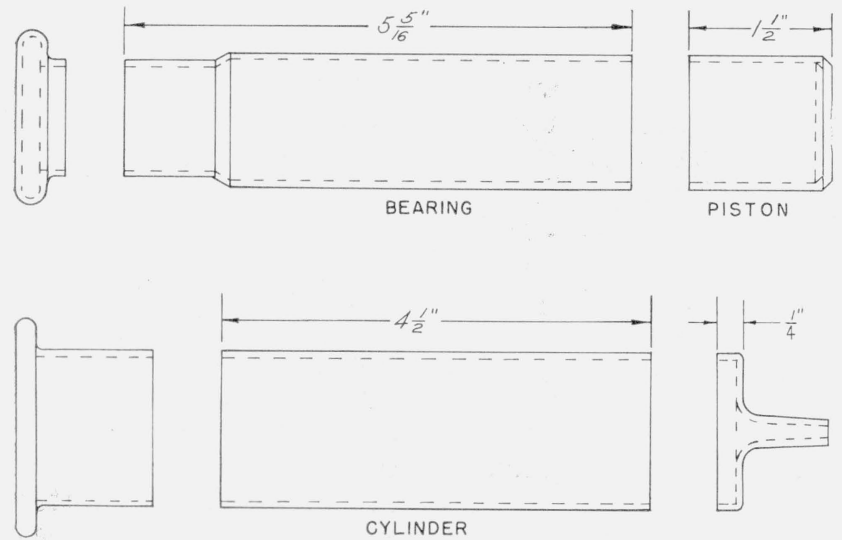

Figure 1. Sawing of hypodermic syringe to obtain parts for piston gage.

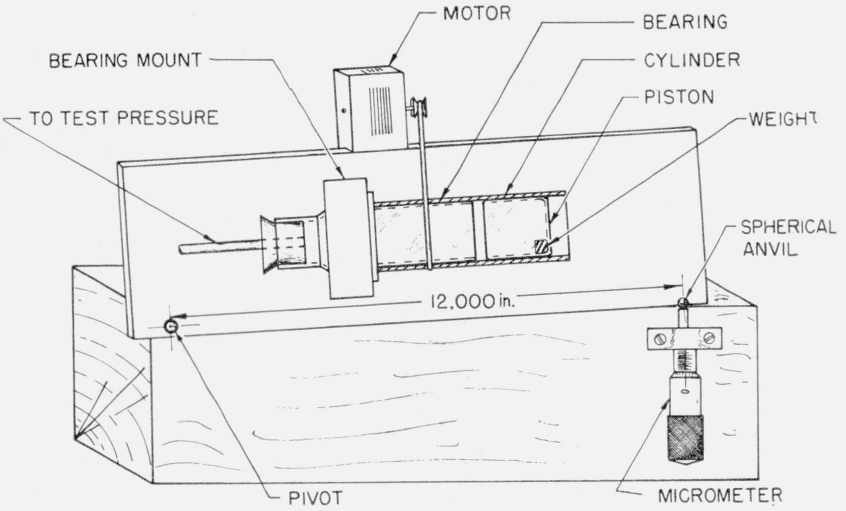

Figure 2. Tilting piston gage, Model $I$.

\subsection{Description of Gage, Model II}

As a result of the experience with Model I, a second gage, Model II, was built. The design was changed so that the tilt can be measured over a 90-deg angle from the horizontal by means of gage end-blocks and 1-in. micrometer caliper. The cylinder on this model was made from 1.250-in. diam precision-bore glass tube. The piston and bearing were prepared, by lapping, from ordinary Pyrex tubing in an attempt to avoid the slight taper that is present in a hypodermic syringe. See figures $3,4,5$. Instead of the wood base of the original model, a $2 \times 6 \times 12$-in. surface plate was used as a base. This provides a satisfactory surface on which to wring the gage blocks used to establish the altitude of the triangle (this being the measure of the tilt). The pivots for the piston support bar and for the micrometer mount are hardened steel cones and spaced $10.000 \pm 0.002 \mathrm{in.}$ apart.

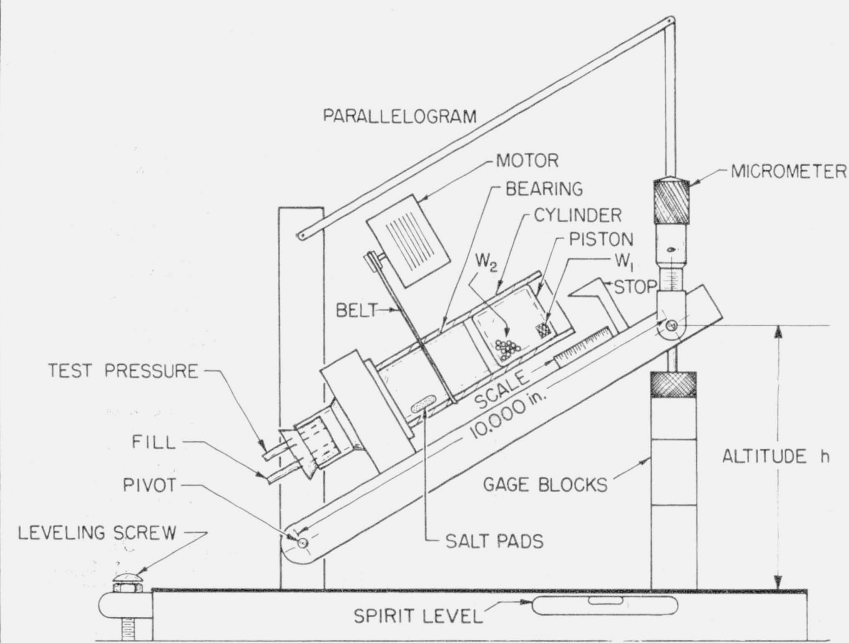

Figure 3. Tilting piston gage, Model II. 


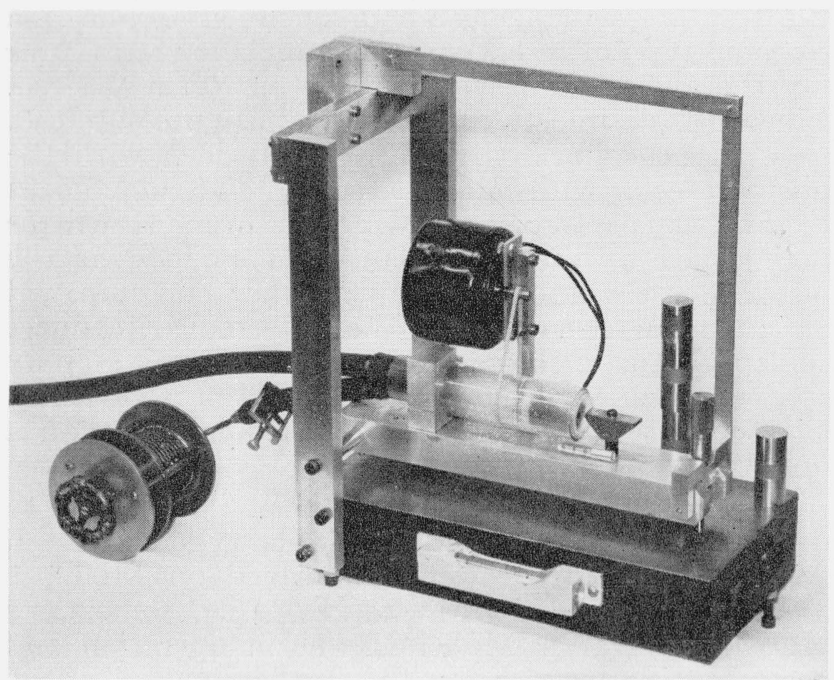

Figure 4. Tilting piston gage, Model $I I$, in zero position.

The micrometer support mount is positioned by a parallelogram structure so that the micrometer axis is maintained perpendicular to the surface of the base plate. The pressure connection is made through the pivot bearing as shown. A stop is provided to prevent the piston being blown out, and for convenience in observing that the level of the base plate is unaltered, a sensitive spirit level is

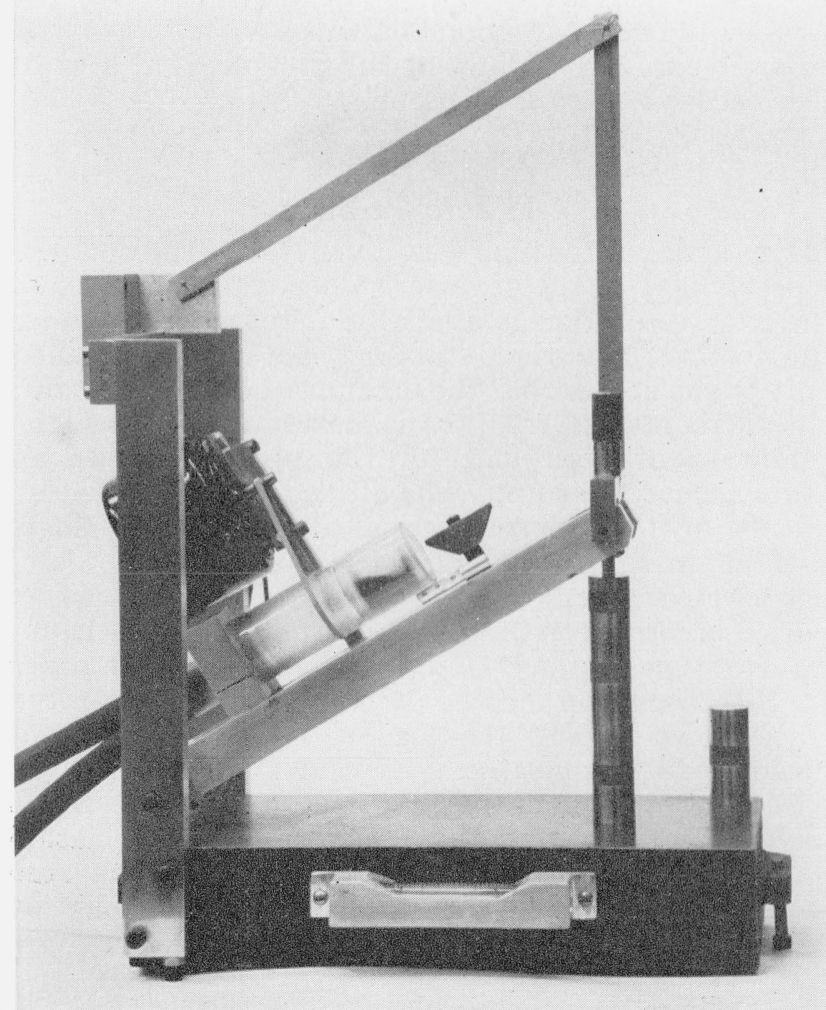

Figure 5. Tilting piston gage, Model II, in tilted position. provided. A small fan motor with a rubber belt provides the rotational movement of the cylinder. The piston is closed with an inside fitted cap and a fixed cast lead weight, $W_{1}$, is used and is advantageously located at the upper end of the piston so that its vertical component will be as nearly as possible through the center of the piston supporting area with a tilt of 3-4 in. In addition to the main weight, $W_{1}$, the piston is loaded with an auxiliary weight, $W_{2}$, composed of lead shot or other such movable weights, the purpose of which will be discussed in section 5.5.

A change in altitude, $h$ of 1 in. is provided by each 1-in. end-block placed under the micrometer and each 1 -in. block is equivalent to 10 percent of the piston weight which is carried by the air pocketed under the piston. The pressure developed by the piston in this pocket is in direct proportion to altitude, $h$. Altitudes up to $8 \mathrm{in}$. have been employed. For altitudes from 8 to $10 \mathrm{in}$. the eccentric position of the weights will not always overcome the cylinder drag and prevent rotation.

The scale length of this model using the combination of the micrometer and end blocks is effectively eight times the scale length of Model I, and thus is readable to 1 part in 80,000 . Some experiments have been made with a 6 -in. diam reading dial such as used on the more precise micrometers. This provides a scale length of $6,000 \mathrm{in}$. readable to $1 / 32$ of an inch and a zero stability of the same order as the resolution.

Two pressure connections to the lower surface of the piston are provided, one for connection to the chamber or space in which the pressure is to be measured and the other, marked "Fill" in figure 3, to replenish air lost by leakage. A controllable volume shown in figure 4 is connected to the "Fill", opening. The function of the salt pad is discussed in section 5.4 .

\section{Calibration}

The pressure difference $p$ between the two end surfaces of the piston is determined by the relation

$$
p=\frac{m g}{a} \sin A=\frac{m g h}{a d}
$$

where $m$ is the mass of the weight; $g$, the acceleration of gravity; $h$, the altitude of the triangle (fig. 3 ); $a$, the effective cross-sectional error of the piston; $d$, the distance between the pivots of the sine bar and calipers (fig. 3 ); $A$, the angle of elevation of the sine bar.

It is seen that $\mathrm{mg} / \mathrm{ad}$ is a constant for any one design, weight of piston and at any one location. When these constants are known, it is only neccssary to measure $h$ to determine the pressure. If the weight $m g$ is in grams and the other quantities in centimeters, $p$ is in units of grams per square centimeter; similarly if $m g$ is in pounds and the other quantities in inches, $p$ is in units of pounds per square inch. 
The piston weight is readily determined, if desired, to 1 part in 100,000 .

In the investigation, relative readings of high precision and reproducibility were desired, but the basic calibration was of lesser concern. Consequently, the precise determination of effective area was not attempted. The effective area has been assumed to be the mean of the cylinder and the piston areas. The diameter of the piston was measured to approximately 1 part in 10,000 by means of a micrometer caliper. The cylinder diameter which is difficult to measure accurately with mechanics' micrometers had an accuracy of about 1 part in 4,000 . Thus the effective area was probably known to an accuracy of about 1 part in 2,000 . Higher precision in this determination would require not only more precise measurement but a combination of piston and cylinder without measurable taper or dimensional irregularities. Meyers and Jessup [4] (as well as later workers) suggest effective diameter determination by manometric methods, and with a suitable manometer available this method would be perferred to clearance measurements. Where a basic gage calibration is required, effective area determination to 1 part in 25,000 for a 1-inch piston should not be too difficult.

There is also some change in area possible, due to internal pressure in the cylinder. Correction for this error may be made from empirical data. And, finally, the effective area may be slightly influenced by the change in the eccentricity of the piston for different angles of tilt. No error from this source has been separated, but some allowance for such an error is indicated in the later tabulation. No dimensional changes have as yet (after 1 year) been recognizable due to either wear or secular changes.

The vertical component of the piston weight is proportional to the sine of the angle of tilt which requires a knowledge of both the altitude and hypotenuse of the triangle (see fig. 3). The leg or altitude of the triangle, measured by the micrometer and gage blocks, can be measured to an accuracy of 0.00005 in. For equal absolute accuracy in the value of the sine of the angle, the hypotenuse must be known to the same accuracy as the altitude.

\section{Performance}

The base of the instrument must be maintained level. Since the resolution is equivalent to the sine of an angle of 2 sec of arc, the axis of the base should be maintained to within at least 1 sec of the horizontal. For highest accuracy, the gage needs to be leveled on a rigid table in a room free from excessive drafts and where the temperature uniformity is such that a thermal expansion error of significance will not exist in either the altitude or area measurements.

The first step is to zero the gage. This is the condition where the pressure connection is open to the room, and the piston in the rotating cylinder is at an equilibrium position touching neither end-stop. Under this condition the axis of the piston is, of course, horizontal. The gage is then connected, to the test pressure, using an independent connection to some type of reservoir to introduce or release air for the repositioning of the piston when desired. When it is desired to provide a definite pressure to a gage to be calibrated, set the altitude $h$ with the micrometer or with micrometer and end-gage blocks to give the pressure desired. This pressure will be maintained at the test connection so long as the piston floats without touching either end-stop. If an unknown pressure is to be determined, connect the apparatus in the same manner but set $h$, step by step, until all axial motion of the piston is canceled. This can ordinarily be accomplished in less than a minute.

Tests of various sorts have been improvised to determine the performance of this type of piston gage. Certain tests were performed on Model I and not repeated on Model II. Also, some tests were devised after Model II was in operation and were not performed on the earlier model. Test results are included irrespective of their being obtained on the earlier or on the more advanced model where they seemed to provide pertinent information on the gage performance.

The readings of the piston gages were compared with those of several sensitive pressure-measuring instruments in order to obtain information on its performance characteristics. These were: (a) An Ascot-Casella micromanometer [3]; (b) a vertical air-lubricated piston gage adapted from a $100-\mathrm{cm}^{3}$ hypodermic syringe; and (c) a sensitive 2 -in. mercury manometer having a null indicator based upon a capacitance bridge [5]. The Ascot-Casella instrument has a torsion balance to measure the difference in the product of the pressure and area of two bells floating on kerosene.

\subsection{Zero Stability}

Particular attention was given to a study of the repeatability of the gage zero since full advantage of the gage resolution is available here, whereas under the loaded condition stability can be determined only to the degree that the load may be held constant. The zero, as referred to, is determined under the condition of both ends of the piston exposed to atmospheric pressure. The altitude $h$ is adjusted by means of the micrometer until the piston floats without axial drift. The repeatability of the zero, determined after altering the former setting by varied amounts, with either intermittent or continuous rotation, has been found to be in the same order as the resolution of the micrometer reading. Six consecutive hourly readings showed variations of +0.0002 to $-0.0001 \mathrm{in}$. in altitude. Daily readings with no rotation of the cylinder during the night have repeated within the same limits for at least 5 days. The influence of other factors on the zero were studied and the results of the tests may be summarized as follows:

(a) There was no visible change in zero with an ambient temperature change of $5^{\circ} \mathrm{C}$. 
(b) A change in the axial position of the piston of $1 / 8$ in. each side of the initial balance point caused a total zero variation of 0.0002 in.

(c) A doubling of the cylinder rotational speed was observed to affect the zero by 0.0002 in.

(d) The piston weight was increased 5 -fold without observable change in the zero.

(e) A piston with an end load of $500 \mathrm{~g}$ was reversed so that the load was positioned first in an outboard position, then inboard position. The average readings, in each position, were identical with a variation in individual readings of only $0.0002 \mathrm{in}$.

Some later tests were made with a 6 -in. dial on the micrometer. Altitude resolution with this micrometer was 0.00005 in. and zero repeatability in tests over several days was to the same limit. With a $30-\mathrm{g}$ piston weight this corresponds approximately to a pressure of $4 \times 10^{-7} \mathrm{in}$. of mercury.

\subsection{Comparison Tests, Pressure Controlled by Tilting Piston Gage}

The first experiments to determine the stability of the reading when the pressure was controlled by maintaining a constant tilt of the piston gage were made with the Ascot-Casella micromanometer as a reference standard. Readings were made on the micromanometer for a period of $20 \mathrm{~min}$ while a constant altitude, or tilt, was maintained on the piston gage during which the axial position of the piston varied $\frac{1}{4} \mathrm{in}$. The micromanometer indications varied approximately 0.2 scale divisions with a full-scale reading of 250 scale divisions. This indicated a repeatability of about one part in 1,000 at a pressure corresponding to about 1 percent of full scale on the piston gage.

Additional comparisons were made in the above pressure range using a hook gage with a resolution of about $0.001 \mathrm{in}$. of water as the reference standard. The repeatability in readings of the piston gage was about the same as with the Ascot-Casella micromanometer. The stability and reproducibility of the zero position of the tilt gage was considerably better than the readings as obtained with either the AscotCasella or hook gage. For this reason it was believed a more reliable reference should be used and subsequent comparison tests were made with the 2 -in. mercury manometer or the vertical air-lubricated gage.

Comparison tests were made at three pressure ranges obtained by varying the weight of the piston. For each piston weight the tilt, or altitude, of the piston gage was varied. In all of these tests the tilting piston gage is acting as a pressure regulator. The pressure readings were made on the reference instrument, in this case the 2-in. mercury manometer.

The results of the low-pressure range comparison are given in table 1 . The check readings were made $5 \mathrm{~min}$ after the first reading. The resolution of the 2 -in. manometer is about $0.00005 \mathrm{in}$. of mercury. It will be seen that the increments for successive increases of 0.2 in. in altitude decrease somewhat as the altitude increases; this increase in sensitivity is not considered significant since the total variation is within the resolution of the 2 -in. manometer.

The results of the comparison at an intermediate pressure range are shown in table 2 . The test was again made on Model I piston gage but with a greater load on the piston. The pressure given in column 2 of table 2 was calculated from the constants of the piston gage and the altitude following the procedure outlined in section 3 . The difference in the pressures calculated for the piston gage and determined from readings on the 2 -in. manometer are given in column 4. The difference amounts to about 1 part in 2,000 of the pressure and is compatible with the resolution of the 2-in. manometer and the uncertainty in the determination of the effective area of the piston.

TABLE 1. Low-pressure range comparison a tilting piston gage Model I and \&-in. manometer

\begin{tabular}{|c|c|c|c|}
\hline Tilting & \multicolumn{2}{|c|}{ 2-in. manometer } & Increments \\
\hline Altitude & $\begin{array}{l}\text { Reading } \\
\text { in. Hg }\end{array}$ & Average & For 0.2 in. \\
\hline $\begin{array}{r}0.2000 \\
.2000\end{array}$ & $\begin{array}{r}0.00160 \\
.00161\end{array}$ & 0.00160 & 0.00160 \\
\hline .4000 & .00320 & & \\
\hline .4000 & .00320 & .00320 & .00160 \\
\hline .6000 & .00479 & 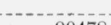 & 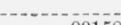 \\
\hline $\begin{array}{l}.6000 \\
.8000\end{array}$ & $\begin{array}{l}.00476 \\
.00633\end{array}$ & .00478 & .00158 \\
\hline .8000 & .00635 & .00634 & .00156 \\
\hline
\end{tabular}

a The reference pressure is $1 \mathrm{~atm}$.

TABLE 2. Intermediate pressure range comparison a tilting piston gage Model I and 2-in. manometer

\begin{tabular}{|c|c|c|c|}
\hline \multicolumn{2}{|c|}{ Tilting gage } & \multirow{2}{*}{ 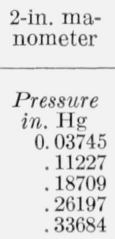 } & \multirow{2}{*}{$\begin{array}{c}\text { Difference } \\
\\
\text { Inches Hg } \\
0.00004 \\
.00004 \\
.00004 \\
.00010 \\
.00015\end{array}$} \\
\hline $\begin{array}{c}\text { Altitude } \\
\text { in. } \\
1.0000 \\
3.0000 \\
5.0000 \\
7.0000 \\
9.0000\end{array}$ & $\begin{array}{c}\text { Pressure } \\
\text { in. Hg calc. } \\
0.03741 \\
.11223 \\
.18705 \\
.26187 \\
.33669\end{array}$ & & \\
\hline
\end{tabular}

a The reference pressure is $1 \mathrm{~atm}$.

The results of the comparison at the highest pressures thus far employed are shown in table 3 . The test was made on Model II piston gage. Again the pressures calculated from the piston gage altitude and constants are compared with the pressures derived from readings made on the 2 -in. manometer. Here the pressures agree within about 3 parts in 10,000 , an amount far less than the expected uncertainty in determining the effective area of the piston. The linearity, which is independent of the piston area, is within about 1 part in 10,000.

TABLE 3. High pressure range comparison a tilting gage Model II and 2-in. manometer

\begin{tabular}{|c|c|c|c|}
\hline \multicolumn{2}{|c|}{ Tilting gage } & \multirow{2}{*}{$\begin{array}{c}\begin{array}{c}\text { 2-in. man- } \\
\text { ometer }\end{array} \\
\begin{array}{l}\text { In. } \mathrm{Hg} \\
0 \\
.28706 \\
.50242\end{array}\end{array}$} & \multirow{2}{*}{$\begin{array}{c}\text { Difference } \\
\begin{array}{c}\text { In. Hg } \\
0 \quad 00005 \\
.00017\end{array}\end{array}$} \\
\hline $\begin{array}{l}\text { Altitude } \\
\quad \text { in. } \\
0 \\
4.0000 \\
7.0000\end{array}$ & $\begin{array}{c}\text { Reading in } \\
\text { Hg calc. } \\
0 \\
.28700 \\
.50225\end{array}$ & & \\
\hline
\end{tabular}

a The reference pressure is $1 \mathrm{~atm}$. 


\subsection{Comparison Tests, Pressure Controlled by an Auxiliary Instrument}

The experimental model of a vertical piston gage was employed at the lowest pressure of its range in a test where the pressure in the system was set and controlled by the vertical piston gage. The altitude of the tilting piston gage, adjusted to bring both pistons to an approximately steady axial position was read at intervals of time. The same point was observed over several hours and repeated the following morning. The results are shown in table 4 .

TABLE 4. Comparison of tilting air-lubricated a piston gauge with a vertical air-lubricated piston gauge

\begin{tabular}{|c|c|c|c|}
\hline \multirow{2}{*}{$\begin{array}{c}\begin{array}{c}\text { Elapsed } \\
\text { time }\end{array} \\
\\
h r \\
0\end{array}$} & \multicolumn{2}{|c|}{ Tilting gage } & \multirow{2}{*}{ 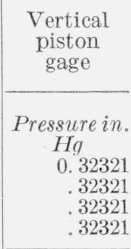 } \\
\hline & $\begin{array}{r}\text { Altitude in. } \\
8.6535 \\
8.6535 \\
8.6545 \\
8.6537\end{array}$ & $\begin{array}{c}\text { Pressure in. } \\
\text { Hg calc. }\end{array}$ & \\
\hline Average....... & 8.6538 & 0.32313 & 0.32321 \\
\hline
\end{tabular}

a The reference pressure is $1 \mathrm{~atm}$.

The maximum departure in the altitude reading of the tilting piston gage from the average was 0.0007 in. or less than 1 part in 10,000 . The difference between calculated pressures as determined by the two gages is on the average $0.00008 \mathrm{in}$. of mercury or 3 parts in 10,000. Again this is less than the expected error in the determinations of the effective areas of the pistons.

This test demonstrated also the ease of balancing the pressures in the two gages by the adjustment of the tilt altitude with the micrometer. The initial balance would be obtained within $4 \mathrm{~min}$ and, after an altitude change of smaller magnitude, balance could be restored in less than one minute. With careful attention and with optical magnification of piston drift a balance can often be effected in one quarter of this time.

None of these comparative tests provided data that shows reproducibility of the reading deflection to the precision anticipated from the performance of the instrument at zero. Although the 24-hr test interval in table 4 showed only a change of two parts in 80,000 , one intermediate reading in the series showed a departure of five times this. An imperfect regulation of the test pressure could be the cause for such variations. In another test Model I tilting gage set at two arbitrary heights, was used as a pressure regulator and Model II was repeatedly adjusted to this pressure. The data are given in table 5 . Here the reproducibility of the setting is 1 part in 25,000 .

\subsection{Off-Center Loading of the Piston}

The effect on the pressure generated by the tilting piston by off-center piston loading was investigated. The center of gravity of the piston for small angles of tilt may be readily positioned over the approxi-
TABLE 5. Repeatability test on Model II, Model I performing as a regulator a

\begin{tabular}{|c|c|c|}
\hline $\begin{array}{c}\text { Elapsed } \\
\text { time }\end{array}$ & Model I & Model II \\
\hline $\begin{array}{r}\text { Minutes } \\
0 \\
40 \\
125 \\
0 \\
5\end{array}$ & $\begin{array}{r}\text { Altitude in. } \\
0.900 \\
.900 \\
.900 \\
4.0 \\
4.0\end{array}$ & $\begin{array}{r}\text { Altitude in } \\
0.6038 \\
.6038 \\
.6038 \\
2.5587 \\
2.5588\end{array}$ \\
\hline $\begin{array}{l}10 \\
15 \\
30 \\
55\end{array}$ & $\begin{array}{l}4.0 \\
4.0 \\
4.0 \\
4.0\end{array}$ & $\begin{array}{l}\text { 2. } 5587 \\
\text { 2. } 5587 \\
\text { 2. } 5588 \\
\text { 2. } 5588\end{array}$ \\
\hline
\end{tabular}

mate center of the load supporting area. However, wide variations are desired in tilt, say up to $75^{\circ}$ or $80^{\circ}$ from the horizontal, in which case the center of gravity of the piston may not even lie directly above any portion of the bearing support area. To test for this effect the piston was end loaded with lead weights and the tilting piston gage connected to the 2 -in. mercury manometer. The altitude of the tilting piston gage was set to 7 in., producing a pressure of approximately $0.225 \mathrm{in}$. of $\mathrm{Hg}^{\text {. The }}$ difference in height of the two mercury columns of the manometer was observed, first with the piston inserted into the tilting gage with the piston weight at the elevated end, and then with the piston reversed end-for-end. This procedure was repeated several times.

The difference in the readings of the mercury manometer, for the orientations of the weighted piston in the tilting piston gage, corresponded to 0.000125 in. in altitude in the 7 -in. deflection. This is less than 1 part in 50,000 and is about equivalent to the least reading of the null indicator of the mercury column.

\section{Other Factors Affecting the Performance}

A number of factors are considered which may affect the satisfactory operation of the piston gage and in some measure may contribute to errors in pressure readings.

\subsection{Maintenance}

The maintenance to keep this gage in excellent calibration and operating condition has been of very minor character. The maintenance so far required has been an occasional cleaning of the piston and cylinder. Cleaning has usually been at intervals of at least 1 month. As there is no wear on the gage itself in use, the precision parts should be expected to have a long life.

\subsection{Temperature}

The most significant error believed present is that due to the change in effective area of the piston through the thermal expansion of the materials of 
which they are made. For Pyrex glass the thermal expansion affects the area less than one part in $100,000 /{ }^{\circ} \mathrm{C}$, for which an approximate correction is readily applied if necessary. If wide temperature variations are anticipated and the highest accuracy is required, the material in the sine bar configuration should, of course, have the same temperature coefficient as the gage blocks.

\subsection{Low-Pressure Limits of Operation}

Only exploratory tests have been conducted at reduced absolute pressures. In these tests the entire piston gage was placed in a pressure chamber and usually the piston gage has been at its zero position.

While the results of these tests indicate the possibility of operation of the piston gage at lower absolute pressures than anticipated, greater difficulty has been experienced in the dissipation of electrical charges on the piston. The use of the salt pad to control the humidity was ineffective. A conductive piston and cylinder might be a solution to this problem but fabrication of these elements was not undertaken. In lieu of conducting materials several "antistatic" agents have been employed. For example a very thin film of sulfuric acid on the glass surface has given completely satisfactory operation to a pressure of a few $\mathrm{mm}$ of mercury for at least an hour. Also, but with an accumulating electrical charge, the piston gage has operated at zero position for several hours at an absolute pressure of below $0.01 \mathrm{~mm}$ of $\mathrm{Hg}$ and at $0.0025 \mathrm{~mm}$ of $\mathrm{Hg}$ for more than an hour. Remarkably there was no indication or suggestion of "lubrication" failure.

The axial motion of the piston at very low pressure becomes very sluggish. The rotational drag on the piston produced by the rotating cylinder first decreases as the pressure is lowered, reaching a minimum at about $2 \mathrm{~mm}$ of $\mathrm{Hg}$, then as the pressure is lowered further there is a rapid increase in the drag which remains about constant to the lowest pressures reached. A "coefficient of friction" calculated for this lowest pressure was 0.4 as compared to a value of only 0.025 at the $2 \mathrm{~mm}$ abs pressure and 0.1 at atmospheric pressure.

At low pressures the static and kinetic friction appear to be about equal, differing from the case at atmospheric pressure where it is sometimes necessary to start the motor repeatedly to secure cylinder rotation without spinning the piston.

The piston had been in use for many hours, thus wearing off the high spots, before the low-pressure tests were made, and the continued smooth operation at low pressure was only obtained when the film of sulfuric acid was present. Without the acid film it was not ordinarily possible to keep the piston in a floating condition at pressures below $1 \mathrm{~mm}$ of Hg. This beneficial behavior of the acid film may be due to its action as a lubricant or to its power to reduce formation of electrical charges or both. The effect of electrical charges on the axial control of the clean piston was estimated to be as much as 20,000 times greater than the effect when the glass surfaces were acid treated.

The air lubrication of the close-fitting bearings of the piston gage particularly at low pressures, warrants further discussion. The horizontal air piston may be regarded as a journal bearing to which the classical hydrodynamical theory of lubrication applies. Computations based on Sommerfeld equations as given by Hersey [6] indicate the load bearing capacity to be at least $10 \mathrm{~g} / \mathrm{cm}^{2}$, more than adequate and the calculated coefficient of friction is a few percent, which is in reasonable agreement with experience.

However, a number of the factors involved in the operation of this instrument are not so easily treated by theory. The Sommerfeld equations assume that the bearing fluid is incompressible and Newtonian, with a definite viscosity. At atmospheric pressure, with a bearing load of the order of $5 \mathrm{~g} / \mathrm{cm}^{2}$ the assumptions of incompressibility would be expected to apply fairly well. At reduced pressures, however, the compressibility of the gas becomes important. To support the bearing load the pressure below the piston must exceed that above the piston by some 3 to $5 \mathrm{~mm}$ of $\mathrm{Hg}$. At $1 \mathrm{~mm}$ of $\mathrm{Hg}$ ambient pressure the gas must, in the crevice below the piston, be compressed by several times and the Sommerfeld theory would be expected to break down. Also when the mean-free path of the gas becomes comparable to the clearance the phenomenon of slip would become important. This will also occur at ambient pressures of the order of a millimeter of $\mathrm{Hg}$. Down to these pressures there is only a slight variation of viscosity with pressure and so there should be very little difference in the coefficient of friction from this source.

At the very low ambient pressures of a few microns of $\mathrm{Hg}$ the ratio of load pressure to ambient pressure and also the ratio of mean free path to clearance are of the order of 100 or a thousand to 1 and the classical lubrication theory would not be expected to apply. An attempt was made to setup the theory of lubrication for the case in which the mean-free path was very large in comparison with the clearance. This bogged down because of the geometrical complexity but it was possible to see resemblances to the classical theory.

The direction of eccentricity makes an angle with the direction of the load which is on the order of 90 deg. The rotation of the cylinder sets up a drift which forces gas into the converging wedge, building up a pressure which will support the load. In the classical theory the gas escapes from the wedge by the viscous flow, in the molecular case the escape is by diffusion. Qualitatively the two cases are similar, but with a different dependance on the local clearance. It appears that at least part of the load can be supported by hydrodynamic lubrication even in the range of molecular flow.

Another mechanism might be expected at the lowest pressures. The treatment with sulphuric acid probably leaves the glass with a large content of water which might out-gas for a long time when 
first evacuated. Also the surface of the glass may be somewhat more slippery than when it is quite clean.

The preliminary considerations of theory are quite obviously raising more questions than can now be answered and it is clear that there is room for more study.

\subsection{Force From Static Charges}

In the earlier use of the gage a sluggishness in the response of the piston was occasionally observed. This was connected with the phenomenon reported by Brubach [2] who states "Some of the syringes had a tendency to center themselves, that is, when the plunger was stabilized at a given point and then pushed in or out of the barrel, the plunger would return approximately to the original point of stabilization." It was suggested in his paper that this action is a function of the asymmetry of the cylinder or piston. However, this phenomenon was believed due to the accumulation of static charges on the piston and was soon connected with changes in the room humidity. The sluggishness was usually present when the relative humidity was lower than 50 to 55 percent and always absent at higher humidities. A simple method of elimination of this trouble was through humidity control. This was readily accomplished by inserting in the hollow bearing of the tilting gage a small blotting paper disk soaked in saturated sodium chloride. This maintained the required humidity for several weeks without rewetting. Since the remedy for this trouble was so simple an exhaustive study of the phenomenon was not made.

The entire force from the electrical charges on the piston when at rest axially, seemed radial and no error in the controlled pressure was observed. When piston motion is required to counterbalance any volume change, the restraining force may, for a few seconds, be a substantial proportion of the piston weight but entirely disappears in 20 to 30 sec if a new position is established. In effect it is as though a considerable amount has been added to the inertia of the piston but nothing to its weight. The piston behaves as though it had a frictionless spring control on its axis with the spring support attached to a frictionless dashpot.

\subsection{Piston Oscillations}

Usually the piston operates with a slight rotational oscillation of several degrees; sometimes the oscillation is entirely absent, but at other times, with some condition critical, such as tilt, piston weight, or rotational speed, the oscillation of the piston increases in amplitude until the piston weight carries over the top. When this occurs the piston continues to rotate at the speed of the cylinder and the gage is inoperable. In the search for some history of this phenomenon a letter of Professor Ott, Ohio State University [7], relative to the failure of the Hackensack bascule bridge which he likened to "a pendulum suspended from a rotating shaft" was unearthed as describing a similar situation.
In his discussions, Professor Ott states "for low speeds the friction decreases rapidly with speed, and the relative motion being greater during the back than during the forward stroke, the angular momentum imparted during the forward stroke would be more than that taken away during the back stroke" and consequently the amplitude of the oscillation increases. This analysis was applied to an oil-lubricated bearing in the reference but would appear to similarly explain the encountered oscillation on this air-lubricated device.

It was found that internal damping in the piston could be used to control the oscillation over a wide range, so instead of using only the eccentrically positioned cast lead weight, loose lead "bird shot" were added to approximately 25 percent of the total piston weight. The movement of the shot on small oscillations is sufficient to damp out any progressive increase in their amplitude.

These oscillations limit the highest pressure that can be reached with the tilting gage. With Model II about 0.5 in. of $\mathrm{Hg}$ can be developed at a tilt angle of about $60^{\circ}$ at $400 \mathrm{rpm}$ of the cylinder with maintenance of good piston stability. Higher pressures should be possible with closer fitting and greater precision in the cylinder surfaces. A recent loading with tungsten carbide granules instead of lead has allowed an angle of tilt of $80^{\circ}$ because of higher damping and a lower center of gravity due to the higher density of the material.

\subsection{Centrifugal Force}

As the cylinder rotates some rotational spin is imparted to the air in the cylinder. This spinning force increases with increase in the rotational speed of the cylinder or with increase, longitudinally, in the distance between the piston and the bearing which would expose more of the cylinder area to the enclosed air. The effect of this centrifugal force was investigated by increasing the space between the piston and the bearing from $1 / 16$ in. to $5 / 16$ in., reading any change in altitude with the micrometer. The altitude change was +0.0001 in., which is the least reading of the micrometer. As an additional check the rotational speed of the cylinder was advanced from $200 \mathrm{rpm}$ to $600 \mathrm{rpm}$. The reading of altitude at the higher speed was within 0.0001 in. of the lower. It was concluded from these tests that any pressure developed by this centrifugal force under normal conditions of operation was without significant effect.

\subsection{Pressure Variations Due to Dynamic Sources}

The piston gage itself is not sensitive to highfrequency fluctuations in pressure but under certain circumstances the fluctuations created by the piston gage may objectionably affect the connected apparatus. These fluctuations can arise from three sources; first, seismic motions, horizontal or vertical; second, any axial component of symmetrical character imparted by the cylinder rotation; and third, from the mechanism used to make up losses in the 
enclosed volume. Such an effect in the order of $2 \times 10^{-5}$ in. of $\mathrm{Hg}$ has been found when following the deflections of a sensitive diaphragm with light fringes. Effects exceeding one light fringe have been observed. These fluctuations have been satisfactorily controlled by connecting the gage to the diaphragm with some damping interposed such as small bore tubing.

\subsection{Leakage}

Leakage from the enclosed volume back of the piston may take place past the piston and the bearing since both are air-lubricated. The test made on Model I with a very low rotational speed while in a vertical position showed a leakage of air of $0.5 \mathrm{~cm}^{3} / \mathrm{min}$ at a differential pressure of $0.3 \mathrm{in}$. of $\mathrm{Hg}$. The leak decreases to zero, of course, when the piston gage axis is horizontal. This leakage is usually obscured by the pumping which occurs.

\subsection{Pumping}

The volume enclosed by the piston is subject to change due to pumping through the air bearings while the cylinder is rotating and may be either into or from the atmosphere. Usually the rate of volume change exceeds the rate of leakage. In the applications for which this gage is being developed the actual loss or gain of volume by leakage and pumping is not significant. When the piston gage is used either as a manometer or as a pressure regulator, the reading has been proven independent of the piston position over a range of $5 / 16$ in., also little manual manipulation is required to maintain the piston position constant to within even $1 / 16$ in. by venting or admitting air in some manner, such as by the adjustment of the screw compressed bellows illustrated in figure 4. It has been found practical to control an admitting valve by a photocell sensing the piston position. Such a controller may readily be added so the chief concern has been to determine whether any error is introduced in the gage readings by any axial forces acting on the piston produced by the pumping or variation in the pumping.

Various influences on the pumping rate were investigated. The pumping action was generally assayed by closing off the line into the gage, observing the axial motion of the piston over a suitable time, usually $5 \mathrm{~min}$, and converting the linear motion of the piston into an equivalent gain or loss in gas volume in the closed space. The results of some of the tests are given below:

(a) With a $90-\mathrm{g}$ piston at zero altitude the pumping rate was, for instance, $0.4 \mathrm{~cm}^{3} / \mathrm{min}$ loss in volume, With other conditions of piston loading, belt tension, or altitude, the rate might be up to $1 \mathrm{~cm}^{3} / \mathrm{min}$ and as frequently a gain in volume as a loss.

(b) The speed of rotation of the cylinder had but slight effect on the pumping rate within the limits tested. Practically identical rates were obtained with a four to one range in the speed of cylinder rotation. Even reversing the direction of rotation by running with a crossed belt, in various trials, gave the same rate of pumping, and in the same direction, as before. This seems to reduce the possibility of the pumping being due here to any screw conveyor action from surface marks or scratches on the cylindrical surfaces.

(c) The weight on the piston had a large influence on the pumping rate. At normal speed and with a piston weight of $30 \mathrm{~g}$ a gain of $0.2 \mathrm{~cm}^{3} / \mathrm{min}$ was noted; and under the same conditions but with a piston weight of $150 \mathrm{~g}$ the rate of gain was $2.0 \mathrm{~cm}^{3} / \mathrm{min}$.

(d) A test was made in which the belt tension was progressively increased. Since the belt pull was upwards on the cylinder and positioned near the outboard end of the bearing, tightening the belt would decrease the bearing load and would at a certain point approximate an equally distributed loading. Under the conditions where light belt tension gave a pumping rate of $1.0 \mathrm{~cm}^{3}$ loss per minute the gradual increase in tension passed through a condition where there was no pumping and on to where the rate was an $0.4 \mathrm{~cm}^{3}$ gain per minute. The increase in belt tension did not result in an observable tilt of the piston axis.

(e) The piston was allowed to rotate with the cylinder by sealing it to the cylinder with a drop of machine oil. The rate of volume gain was found unchanged, indicating no pumping through the piston-cylinder clearance.

(f) The load in the piston was now arranged as unsymmetrically as possible. A $116-\mathrm{g}$ end load was used, arranged so that the center of gravity was slightly off any portion of the piston area. On making tests with this weight in both the outboard and inboard positions the same rate of pumping was obtained in each case, $0.04 \mathrm{~cm}^{3} / \mathrm{min}$ loss. Also as noted previously under zero stability the identical zero was obtained with both configurations. The effect of the unbalanced loading of the piston on the readings, as previously noted, was negligible.

(g) With a piston load of $116 \mathrm{~g}$ and the pumping in a direction to increase the air volume in the cylinder, the tilt of the gage was increased until the leakage rate and pumping rate were identical. Under this condition the pressure in the gage corresponded to 0.1 in. of mercury.

This series of tests seemed to prove that the pumping is chiefly through the cylinder-bearing clearance. Even if some pumping does occur through the piston-cylinder clearance, the magnitude of this effect is less than the resolution of the gage.

A more refined study may be attempted when opportunity is presented in order to determine if, under any conditions not yet covered, there is a measurable axial component reacting on the piston.

Hints as to the mechanism of the pumping are presented in some references on oil lubrication. McKee and McKee [8] studied the distribution of oil pressure in journal bearings and in their eccentric loading test show that the maximum pressure line in the bearing is askew with respect to the axis of the bearing. Also, considerably later, F. W. Ocvirk [9] reported on axial and twisted misalinement as affecting oilpressure distribution in a similar bearing and clearly illustrated with three-dimensional graphs the askew maximum pressure area under these load conditions. 
Neither author discusses transfer of oil through the bearing, but it does seem obvious that if the maximum pressure line (due to dynamic lubrication forces) is askew to the axis of the cylinder the minimum clearance line is also askew, although possibly not in the identical position, and the combination of an askew pressure line followed by a parallel minimum clearance line creates the condition for an axial flow of the lubricant. It is likely that some bell-mouthing also exists to accentuate the off-axis conditions. The conditions causing this askew pressure line, that is, eccentric loading, axial and twisted misalinement, and bell-mouthing (the latter occurring because of the former three) are all present to a much greater degree in the bearing than on the piston. This seemingly bears out the empirical evidence associating the pumping with the bearing. This analysis also suggests methods for reducing the pumping. One method would be to concentrically load the bearing, possibly by the addition of a second bearing or by arranging the bearing to lie outside of the cylinder. A second method would be to lessen twisting misalinement forces by having the rotational drive placed advantageously. Reduction of these latter forces will decrease the likelihood of bellmouthing in use.

This pumping action is of limited importance in the piston gage. The considerable discussion presented here arises because of the difficulty encountered in the attempt to discover if any errors in pressure measurement arise from this action. The primary cause of the difficulty is the fact that the gage has a resolution some 10 -fold greater than any conveniently available instrument on which an error from this source could be evaluated by comparative testing.

\subsection{Gage Volume}

In apparatus where a small constant volume system is required or desired the volume of this gage may be excessive, particularly when combined with the gage leaking and pumping that have just been discussed.

The motion of the piston can be observed, if desired, by low-power optical magnifying means and the position change corresponding to the gain or loss of $0.05 \mathrm{~cm}^{3}$ is reasonably visible, but the volume change of the gage is always significant when there is a movement of the piston. At differential pressures less than $0.1 \mathrm{in}$. of $\mathrm{Hg}$, the increased volume may be contaminated by "in" pumping from the atmosphere in which the gage is operated.

\subsection{Centrifugal Force on the Piston}

An axial force on the piston will exist if the cylinder rotates with any eccentricity. If eccentric motion is imparted to the piston, an axial component may result, affecting the apparent mass of the piston. The construction employed, where the same straight bore of the cylinder is used both to position the cylinder on its bearing and to support the piston, is believed to reduce the eccentricity error to a very low value. No error from this source on either of the models has been detected by change in zero with change in cylinder rotational speed.

\subsection{Summary of Accuracy Limitations}

The limitations upon the accuracy introduced by all factors are collected and summarized in table 6 . Where dependent upon deflection, the values listed may be assumed for a tilting gage set at an altitude $h$ of 5 in. and having a medium-weight piston. Many of these listed values are not known to sufficient precision to establish their variation with gage deflection. Values have been assigned in various cases to represent what is, at present, believed to be the maximum error that would be contributed by the listed factor. In many cases the value of 0.0001 -in. is assigned because this represents the resolution of the gage reading at the time of the error investigation. Some of these errors may not even be of this significance. A few of the values entered are lower than this since they were studied when higher resolution was available for the reading.

Table 6

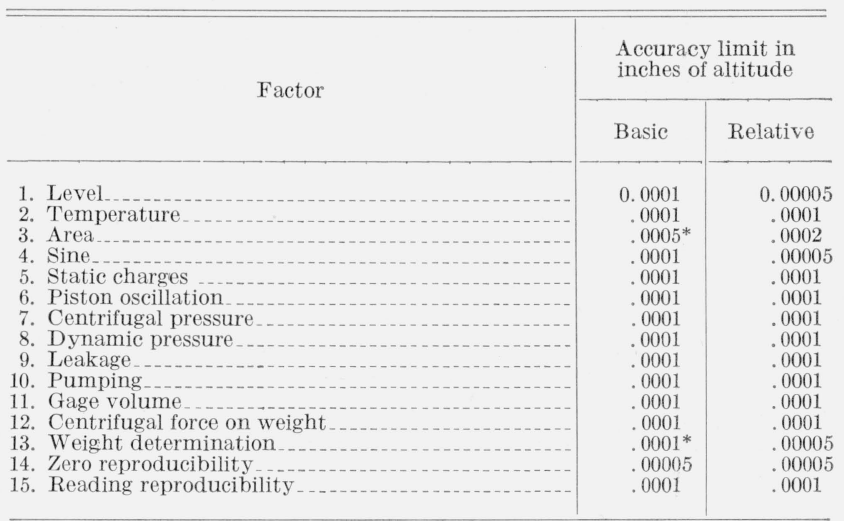

In the error columns the values under "Basic" are assigned to cover the error of the instrument as a primary standard. In the column under "Relative" the values are based on the ability of the gage to repeat a reading either as a gage or as a pressure regulator. The errors without asterisks are subject to variations dependent on random influences. The errors marked with the asterisk are unidirectional. The 5-in. altitude with the medium-weight piston corresponds to a pressure reading of about $0.4 \mathrm{in}$. of $\mathrm{Hg}$. The maximum predicted error in the basic measurement is $8 \times 10^{-5} \mathrm{in}$. of $\mathrm{Hg}$ and in the relative measurement is less than $4 \times 10^{-5}$ in. of $\mathrm{Hg}$.

\section{Design Modifications}

Although because of its simplicity, an air-lubricated bearing has been employed for the cylinder, this is by no means essential and does have the inconvenience of considerably increasing the exchange between the atmosphere within and without the gage. Oil-lubricated journals or ball bearings 
could be employed and the pressure chamber sealed with O-rings.

Where it is desired to work from a pressure datum other than that of the room, this may be accomplished by several means. For instance, identical air-lubricated bearings for the cylinder may be employed at both ends of the piston. This has been done at times, but a very nice mechanical assembly is required to allow disassembly for cleaning or adjusting the piston weight. If self-alinement ball bearings were employed this need not be a difficult reassembly.

Alternately, a particularly compact structure could be made by making the outer surface of the cylinder the bearing face, concentric with and outside the piston support area and fitted with two end pressure taps, as shown in figure 6 . Alternatively the whole device may be enclosed by a belljar or to insure ease of access to the micrometer for adjustment, the cylinder section only might be enclosed and driven through a magnetic coupling.

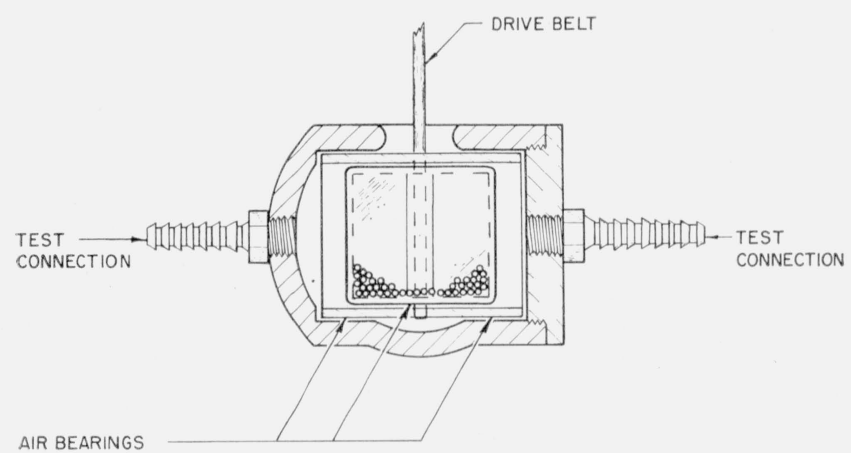

Figure 6. Modified piston gage.

Symmetrical pressure connections are provided to control the reference pressure also. The bearings are outside of the cylinder.

If the reference pressure is to be a high vacuum, the highest precision would be necessary in the cylinder fitting. Even with the smallest working clearance between the piston and cylinder the approach to the reference pressure would presumably be limited by the leakage past the piston, and the operation would be limited to an average pressure that would allow an air film for lubrication of the piston.

For a reference standard, it is believed that the most desirable piston and cylinder combination would be of fused quartz because of the stability of this material and also because of its nice working qualities. The fitting of the piston to the cylinder and area measurements might be performed to a much higher order of accuracy than with glass. Transparency of the cylinder seems desirable for observation of the piston although for a structure such as figure 6 an end window would suffice, in which case the cylinder and piston could both be metallic. It is desirable for the piston and cylinder to be of identical materials to avoid temperature effects in the close fits required.
To reach the lowest pressures the piston weight should be the least possible. No attempt has been made to determine the optimum diameter of the piston. A diameter on the order of $1 \mathrm{in}$. is convenient to work with, but smaller diameters might be employed with equal satisfaction if the volume or leakage of the gage is particularly significant. The eccentric weight for the piston may be, on various arrangements, suspended outside of the cylinder in order to provide greater leverage if needed.

The micrometer or height setting means might be driven by a servomotor to maintain a null piston position. Increased resolution is available if the micrometer were provided with a 6 -in. reading circle or a digital readout, and provided that the screw has commensurate accuracy.

\section{Field of Application}

Because of its sensitivity and inherent accuracy it is believed that this gage has application as a calibrating standard in the range of differential pressure up to at least $1 / 2$-in. of mercury and possibly can be extended to twice this. Its aperiodic motion and low-temperature coefficient (particularly if of quartz) reduces the time for a calibration.

Tests at zero differential pressure but at low absolute pressure indicate that measurements can be made at absolute pressures down to a few millimeters of $\mathrm{Hg}$. With further development, it appears possible to extend the lower pressure limit down below a 0.1 of a millimeter of $\mathrm{Hg}$.

As a manometer to read an unknown pressure in the above range, it has considerable versatility and with its dead-beat characteristics allows readings to be obtained rapidly. As a pressure regulator to reproduce precise settings, it has the unique advantage of the commonly used dead weight tester.

\section{References}

[1] W. G. Brombacher and T. W. Lashof, Bibliography and index on dynamic pressure measurement, NBS Circ. 558 (1953).

[2] H. F. Brubach, Some laboratory applications of the low friction properties of the dry hypodermic syringe, Rev . Sci. Inst. 18, 363 (May 1947).

[3] H. R. Hindley, A differential manometer, J. Sci. Inst. 24, 295 (1947).

[4] C. H. Meyers and R. S. Jessup, A multiple manometer and piston gage for precision measurements, BS J. Research 6, 1061 (1931) RP 324.

[5] Manometer for calibrating high-altitude pressure devices, NBS Tech. News Bull. 43, 71 (1959).

[6] M. D. Hersey, A short account of the theory of lubrication, J. Frankl. Inst. 220, 93 (1935).

[7] P. W. Ott, Letter to Eng. News Rec. 103, 785 (1929),

[8] S. A. McKee and T. R. McKee, Pressure distribution in oil films of journal bearings, Trans. Am. Soc. Mech. Eng. APM-51-15, 161 (Nov. 1931).

[9] F. W. Ocvirk, Measured oil film pressure distribution in misaligned plain bearings, Lub. Eng. 10, 262 (Oct. 1954).

Washington, D.C.

(Paper 63C1-5) 